CAHIERS DE

NARRATOLOGIE

\section{Cahiers de Narratologie}

Analyse et théorie narratives

32 | 2017

Récit et argumentation, interactions, lieux et dispositifs sociaux

\title{
Récit, preuve et témoignage : argumenter en histoire à l'école
}

\section{Sylvain Doussot}

\section{(2) OpenEdition}

Journals

Electronic version

URL: http://journals.openedition.org/narratologie/7830

DOI: 10.4000/narratologie.7830

ISSN: 1765-307X

Publisher

LIRCES

Electronic reference

Sylvain Doussot, " Récit, preuve et témoignage : argumenter en histoire à l'école », Cahiers de Narratologie [Online], 32 | 2017, Online since 21 December 2017, connection on 02 May 2019. URL: http://journals.openedition.org/narratologie/7830; DOI : 10.4000/narratologie.7830

This text was automatically generated on 2 May 2019.

Article L.111-1 du Code de la propriété intellectuelle. 


\title{
Récit, preuve et témoignage : argumenter en histoire à l'école
}

\author{
Sylvain Doussot
}

1 Si la force des liens entre narration et argumentation dans le champ de l'histoire est bien connue, tant dans le grand public que dans le monde de la recherche, la connaissance de leur dimension problématique n'est pas aussi bien partagée. Car si l'audience des travaux historiques au-delà des sphères académiques repose notamment sur la forme narrative dans laquelle elle s'exprime, c'est cette même forme narrative qui, en confondant histoires et histoire (que l'anglais distingue en stories et history), questionne la scientificité même de la discipline, y compris dans les débats épistémologiques qui animent ce champ de recherche depuis un quarantaine d'années. Ce qui distingue le texte d'histoire scientifique d'autres récits sur le passé se situe essentiellement en deçà du texte, dans les pratiques d'enquête. Celles-ci reposent sur des modalités spécifiques de mise en relation des données empiriques (les traces du passé) et des modèles de comportement humain pour produire des explications (dans telle situation observée, les hommes ont tendance à se comporter de telle manière). L'enquête de l'historien ne conduit pas seulement à des explications vraisemblables des événements et des phénomènes, elle produit les raisons, adressées aux pairs, de penser que l'événement s'est passé d'une certaine manière et non d'une autre, en explorant systématiquement les données pertinentes et les modèles explicatifs possibles. Dès lors, chez les historiens, deux récits s'imbriquent plus ou moins explicitement : le récit de l'événement et le récit de l'enquête sur l'événement.

Dans cette configuration, l'histoire scolaire se trouve placée dans une position délicate. Elle s'ancre depuis longtemps - pour la France tout au moins, mais sans doute au-delà dans une double finalité qui radicalise la dualité entre événements du passé et enquête sur les traces de ce passé : l'enseignement de l'histoire vise aussi bien à faire appréhender et maîtriser par les élèves des récits établis d'événements du passé, qu'à développer chez eux des capacités critiques et argumentatives fondées sur l'étude critique des traces du passé. Il s'agit pour les enseignants de tenir à la fois l'objectif de compréhension et de production de récits descriptifs du passé sous la forme de narrations chronologiques, et l'objectif de production d'une argumentation en vue de prouver, par l'enquête historique 
à la mesure de l'école, que le récit en question est fondé en vérité sur l'étude des traces. Mais les recherches didactiques sur les pratiques ordinaires (Tutiaux-Guillon, 2008) montrent que dans la classe cette dualité tient davantage de la dichotomie que de la dialectique. Elle s'incarne dans la séparation vécue par les élèves entre les phases d'étude de documents (démarche de preuve) et les phases d'écriture de la trace écrite (mise en récit du passé), mais également dans la séparation entre les documents et le passé. Cette position spécifique de l'histoire scolaire renvoie toute recherche didactique à la question historiographique et épistémologique - notamment discutée par Ginzburg (2008) - des rapports entre rhétorique et preuve, entre efficacité du discours de l'histoire et validité scientifique.

3 Dans le champ des recherches en didactique de l'histoire, nous ${ }^{1}$ abordons ces questions selon une approche qui accorde une grande importance aux enjeux épistémologiques disciplinaires, et à la relation entre la discipline scolaire et la discipline scientifique. Nous nous intéressons à ces deux dimensions disciplinaires sous l'angle des rapports qu'entretiennent les savoirs comme textes, et les pratiques qui les rendent possibles, et donc à l'analogie entre ce rapport chez les historiens et dans les classes. Dans un tel cadre théorique, nous proposons (Doussot, 2011) d'appréhender la question de l'argumentation en histoire scolaire, prise dans la dichotomie du récit et de l'enquête, à travers l'hypothèse de son dépassement par la mise en tension des données du passé et des modèles explicatifs du présent, comme processus fondamental de problématisation historique. Une telle mise en tension d'ordre épistémologique se traduit dans l'activité de recherche et d'écriture par la négociation entre récits concurrents (Levisohn, 2010). Ce processus concerne aussi bien les témoignages contemporains de l'événement que les points de vue plus éloignés, y compris ceux des historiens. Une telle négociation constitue un jeu d'échelles (Revel, 1996) caractéristique de la communauté historienne, qui met en discussion les traces laissées par des actions singulières passées et les savoir généraux sur la période et sur les comportements sociaux. A quelle condition serait-elle possible dans le cadre scolaire?

Nous abordons cette question en recensant les récits et les points de vue qu'ils portent dans la classe d'histoire, avant de voir (section 2) que les difficultés liées à cette pluralité se résolvent ordinairement par l'application implicite d'un cadre d'analyse chronologique et causal. Celui-ci tend à empêcher toute remise en cause de la linéarité de l'enquête scolaire sur le passé: les sources donnent des informations sur le passé que l'élève configure dans un récit explicatif, sous le contrôle de sa cohérence avec la suite des événements. Or seule une mise en discussion de cette linéarité peut permettre d'esquisser un jeu d'échelles analogue à celui des historiens. La section 3 engage alors une réflexion comparative sur le travail des sources à l'école et chez les historiens qui met en lumière un principe fondamental de la construction argumentative de ces derniers : à la linéarité d'une appréhension réaliste des sources se substitue une exploration en deux dimensions du champ des possibles de l'action que constitue la source; exploration qui prend en compte les incertitudes des acteurs. L'historien s'intéresse à ce qui aurait pu advenir afin de rendre raison de ce qui est advenu, en reconstituant les choix envisageables par les acteurs de l'événement. Deux exemples scolaires illustrent ces deux perspectives, le deuxième (section 4) montrant une analogie certaine avec les processus scientifiques. La comparaison didactique de ces deux cas conduit (section 5) à identifier les conditions de possibilité du développement d'une enquête historienne scolaire qui sort de la linéarité des explications causales au profit d'un processus de reconstruction du passé à la fois 
configurant et intrigant, qui peut donner vie à une histoire non linéaire de la recherche menées dans la classe elle-même. Parmi ces conditions, l'usage du témoignage comme source intrinsèquement narrative apparaît déterminant.

\section{Récits du passé et points de vue dans la classe}

5 Le récit est un moyen de rendre compte d'événements et de phénomènes, mais un récit est toujours indexé à un point de vue. La littérature, le cinéma, ou plus récemment les séries télévisées l'illustrent parfois de manière explicite en structurant leurs productions sur le jeu de points de vue multiples qui sont autant de récits concurrent d'une réalité insaisissable. De cette pluralité il découle immédiatement une question : quelle est le bon récit? Qui était le mieux placé pour voir l'événement et, par conséquent, pour en témoigner?

En classe d'histoire, de la même manière, se croisent potentiellement plusieurs récits d'un même événement : les récits portés par les documents (des témoignages, des acteurs des événements), et ceux de la classe (du manuel, des élèves, de l'enseignant). Selon l'acuité des événements en jeu, ces différents récits peuvent être plus ou moins influencés par les débats de société qui interfèrent dans le cours de la classe. Mais le récit de l'enseignant est a priori celui qui est le plus légitime, parce que son autorité repose notamment sur son savoir, lui-même fondé sur sa connaissance des récits des historiens. Sous cet angle, pour les élèves, la question du bon récit ne se pose pas ou bien pas longtemps : elle est réglée par la logique autonome de la discipline scolaire (Tutiaux-Guillon, 2009) qui fait du récit institutionnalisé dans la trace écrite qui conclut le cours la référence pour l'évaluation.

7 Un tel fonctionnement ne poserait pas de problème si l'enjeu de l'apprentissage historique se limitait à la connaissance du bon récit. Mais une autre finalité s'ajoute à l'enseignement de l'histoire qui peut paraître en contradiction avec l'établissement et la compréhension d'un récit unique : la capacité critique à évaluer les récits possibles sur un événement, en relation non pas avec la qualité de l'auteur, mais en relation avec le réel porté par les traces de ce passé. C'est la raison pour laquelle le cours d'histoire ne se limite pas à faire écrire et apprendre la trace écrite, mais est la plupart du temps structuré autour de l'étude de documents.

8 Cette contradiction pose un premier problème en mettant en évidence dans le cours de l'activité scolaire qu'il existe plusieurs points de vue possibles. Cela peut conduire à une alternative insatisfaisante : soit le relativisme (tous les récits se valent), soit l'objectivisme (il existerait un bon point de vue qui serait un point de vue surplombant, c'est-à-dire tous les points de vue réunis). Le premier terme de l'alternative constitue un risque avéré par des études didactiques (voir par exemple Wineburg, 2001, chap. 4). L'étude de GerinGrataloup, Solonel, \& Tutiaux-Guillon (1994) montre que des élèves de troisième n'identifient pas de problème dans l'opposition des points de vue portés par différents journaux sur la journée d'émeutes meurtrières du 6 février 1934, du fait de l'évidence pour eux que chacun voit les événements par le prisme de son idéologie, tout au moins lorsqu'il s'agit d'événements "politiques ». Autrement dit, les élèves identifient la coexistence de points de vue variés, mais ils ne maitrisent pas cette variété.

Le second terme de l'alternative, l'objectivisme, est sans doute plus délicat parce qu'il est emprunt d'une conformité avec le sens attribué par les élèves aux pratiques de classe: 
l'étude de documents est là pour soutenir le récit de l'enseignant selon une vision réaliste simpliste qui séparerait le récit historique du récit de fiction. Dans cette évidence scolaire qu'Audigier (1995) qualifie de réalisme, ce ne serait pas l'enseignant (et derrière lui l'historien) qui parlerait, mais par lui la source elle-même. On voit par là que le recours direct aux sources - dans le cadre de l'étude de documents, activité de base du cours d'histoire - ne fait que déplacer le problème. Le recours aux documents est un moyen de ne pas attribuer au professeur la vérité du récit de la classe, mais fonder la vérité du récit sur l'étude de documents passe nécessairement par son interprétation. Or, par effet du réalisme qui fait du document le passé lui-même, son étude scolaire masque l'inévitable interprétation qui est à l'œuvre. Autrement dit, dans cette configuration dominante du cours d'histoire, le point de vue de l'élève ou du professeur sur le document médiatise le point de vue sur le passé lui-même, mais de manière invisible: les grilles de lectures s'imposent aux traces sans qu'il soit possible de les discuter. On retrouve là le mécanisme mis en évidence par White (1978) qui relativise la scientificité des récits des historiens.

L'enjeu d'analyse didactique de l'étude de documents qui structure le cours d'histoire habituel réside donc dans le processus d'interprétation. En particulier, il pointe le rôle essentiel du cadre d'analyse qui préside à l'interprétation et constitue de ce fait le point de vue généralement implicite sur le document.

\section{Un cadre d'analyse chrono-causal et téléologique}

11 Parmi les différents récits qui co-existent dans la classe, deux catégories se détachent plus ou moins explicitement. D'une part, les documents fournissent souvent des récits sur l'événement lorsqu'il est en train de se produire, avec l'idée que la proximité temporelle et spatiale avec l'événement garantit au témoin une plus grande légitimité. Ainsi, qui mieux qu'un soldat ayant combattu dans les tranchées serait en mesure de dire ce qu'était l'expérience de la Grande Guerre? D'autre part, le manuel, le professeur, et les élèves eux-mêmes, fournissent des récits après-coup, en sachant la suite de l'histoire. Ce décalage entre situation étudiée et situation d'étude et d'énonciation est fondamental pour qualifier les différents types de récits en jeu (Bronckart, 1996). D'un côté l'étude de témoignages fournit la caution d'une proximité - une conjonction - entre le texte du témoin et les faits en question. L'enseignant les choisit cependant de telle manière qu'ils soient cohérents avec le récit qu'il vise et qu'ils doivent renforcer. Dans la deuxième phase du travail qui conduit à la trace écrite du cours, au contraire, c'est la disjonction d'avec l'événement qui vaut. L'élève accorde en effet du crédit au récit de l'enseignant non seulement parce qu'il à lu les historiens, mais aussi parce qu'il s'appuie sur une connaissance de la suite de l'histoire. Du fait de cette position, il est capable de produire ses propres récits dans le vif de la classe. Ses explications ne sont pas le fruit de sa fantaisie et de son imagination, mais sont tenues par la série des événements qui ont suivi et qu'il connaît. Ce point renforce l'asymétrie entre le professeur et les élèves. Eux pensent qu'ils peuvent mal interpréter un événement parce qu'ils ignorent beaucoup des événements avant et après celui qui est en jeu. C'est d'ailleurs cette dimension encyclopédique du savoir historique qui est valorisée lorsqu'on attend des élèves des bases et des repères comme prérequis. Ainsi, une des principales activités de l'histoire scolaire consiste pour les élèves à reproduire le savoir déjà vu²

Pourtant, cette caractéristique téléologique des récits historiques scolaires (mais aussi grand public) manifeste une faiblesse épistémologique si on la compare aux récits des 
historiens. Les historiens savent en effet qu'il faut se méfier de "la chimère des origines » : la construction linéaire de l'histoire qui relie sans fin un événement à l'autre en leur supposant des liens de causalité, jusqu'à nous, comme si tout ce qui s'était passé tendait vers notre monde, comme si le cours de l'histoire n'avait pas eu d'autres possibles, des bifurcations qu'on ne voit plus par effacement des voies non suivies. Un tel régime téléologique tend à faire disparaître l'incertitude qui caractérise l'expérience historique des acteurs sociaux au profit de causalités - plus ou moins plurielles et enchâssées - qui rendent inutile le cœur de l'argumentation scientifique : la production de raisons, c'est-àdire d'explications non sur le cours des choses mais sur les bifurcations disparues. En visant à expliquer non seulement ce qui s'est effectivement passé, mais aussi pourquoi il n'en a pas été autrement, le texte de l'historien ne présente pas de causes possibles aux événements sans en évaluer simultanément les conditions de possibilité. Cette caractéristique apodictique $\mathrm{du}$ savoir historique des historiens est celle de l'argumentation scientifique.

Il est possible d'illustrer ce processus par un exemple à propos de la Révolution. La vulgate scolaire fait apprendre l'existence des Lumières et des révolutions anglaise et américaine, avant d'enseigner la Révolution française. Cette simple succession suffit à expliquer l'événement par un lien de cause à effet. Voici par exemple comment une élève de 9 ans parvient, dans un échange avec le chercheur, à produire un récit chrono-causal après une leçon :

- Alors c'est quoi la Révolution, toi qui écoute les $\mathrm{CM}^{3}$ ?

- ... euh, alors en fait c'est un roi qui, euh..., qui..., qui fait payer plein, plein d'impôts à son peuple... pour avoir plein de... richesses euh... et puis il faisait des grandes... des grands festins pour montrer euh... qu'il était très digne, à plein d'autres gens. Puis, euh, les gens ils se sont révoltés. Parce qu'ils trouvaient ça injuste. Et puis euh, après il y a euh... je crois que c'est Louis XIV, je suis pas sûre?

- Non, c'est Louis XVI

- Louis XVI oui ben voilà, il s'est fait guillotiner.

- D'accord. Mais c'était qui ces gens qui se sont révoltés?

- C'était les paysans, le peuple.

- Mais comment ça se fait, le roi il a des soldats comment ça se fait qu'il s'est pas défendu?

- Ben parce qu'ils ont... ils étaient plein, plein, plein ; plein, plein, plein, plein.

- Plus que les soldats?

- Oui.

- Mais euh... ça faisait longtemps qu'ils étaient malheureux, pourquoi ils ne se sont pas révoltés avant?

- Ben parce que il y a quelqu'un, je sais plus qui c'était, qui leur a dit mais laissezvous pas faire, vous avez... vous voyez ce qu'il vous fait, il vous fait payer plein d'impôts, vous pouvez presque plus manger.

- Ah ouais, parce qu'ils ne s'en rendaient pas compte?

- Ben ils étaient habitués.

- Mais il y a quelqu'un qui le savait. Et qui leur a dit.

- Euh, je sais plus qui s'est.

- La maîtresse elle en parlait aux CM2 ?

- Oui... c'était le machin des Lumières

14 La logique du temps qui passe comme support de causalité est bien ancré chez cette élève: le raisonnement post hoc ergo propter hoc lui permet de faire des textes philosophiques des Lumières des causes de la Révolution par application d'un modèle explicatif général (quand on nous montre qu'on est mal traité, on se révolte). Or, écrit l'historien Chartier (2000), on peut inverser le rapport chronologique ordinaire en se 
demandant simplement si l'on parlerait encore et dans ces termes des Lumières si la Révolution n'avait pas eu lieu. D'une manière contre-intuitive, il suggère que l'événement ultérieur a «fait» l'événement antérieur tel qu'on le perçoit aujourd'hui. La logique conjecturale qui émane du problème développé par l'historien s'oppose ici à la chronologie causale de l'élève qui, sans questionnement, fait de l'événement qui suit la conséquence de celui qui précède.

par cet exemple comment les choses se compliquent dans une perspective didactique. Même si la perspective téléologique pose problème, on ne peut d'une part ignorer ce qu'on sait de la suite de l'histoire qui reste, même dans la perspective de l'historien, un principe d'intelligibilité de ce que sont les «Lumières ». Autrement dit, le problème historique évoqué par Chartier ne peut naître que d'une connaissance de l'enchaînement des événements, mais il ne peut pas non plus s'y réduire. Il ne faut donc pas, pour l'enseignement, jeter le bébé des savoirs factuels avec l'eau du bain de la téléologie, mais en revanche, il n'est pas envisageable de se contenter de causes générées par la simple succession des faits.

Ce serait plutôt, chez Chartier comme chez d'autres historiens, la dialectique de ce qui est avéré et de ce qui était possible qui serait productive au sens scientifique du terme. Il est possible de l'envisager de manière formelle, c'est-à-dire en dehors de la complexité du réel de l'enquête historique, par le biais de la littérature lorsqu'elle se risque à la production d'uchronies. Le cas du roman de Philip Roth, Complot contre l'Amérique, en constitue un bon exemple. Il met en scène une famille juive dans l'Amérique des années 1930, celle du président Roosevelt, mais le récit s'échappe de la trame historique réelle lorsque l'auteur choisit de faire perdre Roosevelt à l'élection présidentielle de 1936 au profit du héros aviateur Lindberg, notable soutien de l'Allemagne hitlérienne. Le choix de l'auteur de respecter la réalité historique avant et après la période considérée, le contraint à organiser la partie de son récit qui est écrite sur fond historique fictif d'une manière compatible avec ce que les historiens nous en disent. Dans le roman, le cours de l'histoire bifurque au profit d'une voie possible mais non advenue effectivement, ce qui en fait plus précisément une histoire contrefactuelle si l'on s'intéresse en particulier à l'événement déclencheur que constitue l'élection de Lindberg à la place de Roosevelt. Ce choix oblige l'auteur à faire état des conditions de possibilité de ce qui n'est pas advenu réellement mais fictivement: le début du roman est alors sans doute la partie la plus délicate, qui doit montrer l'Amérique de Roosevelt telle qu'elle était, mais aussi comme pouvant potentiellement bifurquer. D'une certaine manière, l'invention contrefactuelle rend nécessaire un point de vue plus questionnant sur l'événement et son contexte d'émergence, pour rendre vraisemblable le fictif.

17 Le détour par le roman, s'il fait voir le sens de l'opération de reconstruction des voies non advenues, ne peut cependant pas se substituer à l'analyse de la pratique d'enquête qui permet ce processus d'exploration des possibles du passé à partir des traces qui en subsistent. Cette analyse est nécessaire pour dégager les principes qui président à l'enquête historique, afin d'en considérer la possible transposition dans le cadre scolaire. 


\section{La critique des sources comme construction de problème historique}

Reprenons l'exemple de la Révolution française. Dans l'appréhension des origines de la Révolution, les textes des philosophes des Lumières peuvent naturellement constituer des traces en faveur d'une interprétation téléologique qu'on a évoquée : tel texte de Rousseau ou de Voltaire peut être lu aujourd'hui comme précurseur de l'événement, et comme ayant motivé le passage à l'action. Le texte peut alors, en tant que tel, fournir les indices d'une analyse critique de la monarchie qui aurait été le moteur de la révolte. Même si le document est bien souvent "présenté » au préalable - selon un rituel qui demande aux élèves d'identifier l'auteur, la date, la nature, les destinataires etc. - des questions mènent ensuite les élèves à sélectionner des informations ponctuelles. Dans un tel dispositif, les élèves ne contextualisent pas le document au sens où l'entendent les historiens. On ne le leur demande pas parce que c'est justement la construction du contexte qui est en jeu : la logique de l'étude de documents consiste à analyser un document par définition singulier pour en induire le contexte. L'idée explicative implicite de ce travail sur des textes des Lumières est que leur lecture produit un contexte mental favorable à la révolte contre la monarchie. On retrouve là le travers téléologique, mais à un niveau plus subtil. L'analyse en termes de cause et d'effet linéarise inévitablement l'explication du changement et le rapport de l'action, portée par la source, à son contexte. La classe travaille sur les textes des Lumières comme s'ils n'avaient pas de contexte de réception, ou plutôt comme si le contexte de réception était vierge, comme si le Peuple lisait ces textes et comme si les lecteurs n'avaient pas de représentations qui influenceraient leur lecture. Schématiquement, on peut dire que le récit produit par une telle enquête scolaire est le suivant : les Philosophes écrivent une critique de la monarchie absolue, elle est lue par le Peuple, ce qui crée un contexte propice à la révolte qui survient à un moment de fragilité de la monarchie (crise financière).

Or que font les historiens face à ce type de documents? Ils les confrontent à ce qu'ils savent du contexte en s'appuyant sur ce qui est établi par les travaux antérieurs, et dans le cadre d'une critique des sources qui vise à dégager les intentions du document : qui a lu ces textes? Comment ont-ils été lus ? Pour répondre à ces questions, il faut savoir quelles sont les représentations des lecteurs, mais plus généralement, ce que lisaient les futurs Révolutionnaires et ce qu'ils pensaient du roi et de la monarchie. Les historiens poursuivent donc l'enquête initiée par la lecture critique des textes des Lumières : ils identifient la multiplication des libelles, petits livres satiriques, diffusés de plus en plus massivement, notamment à Paris, et qui, dans les décennies qui précèdent la Révolution, participent activement à une désacralisation du roi et de son entourage (Chartier, 2000).

Ce résumé très succinct schématise un processus de problématisation historique, c'est-àdire un processus qui part des savoirs stabilisés, en questionne les fondements dans les traces (les textes philosophiques) en les prenant non pour des textes mais pour des actions (les textes dans leur valeur performatrice : ce qu'ils font à ceux qui les lisent), ce qui conduit vers d'autres traces (les libelles), et ainsi de suite. Ce processus permet de faire émerger les conditions de possibilité que les textes des philosophes soient considérés comme des causes de la Révolution (hypothèse de départ): les textes philosophiques n'ont de valeur explicative qu'en relation avec un mouvement plus profondément diffusé de désacralisation de la monarchie. 
21 Sous cet angle, la différence essentielle entre le processus de problématisation scientifique des historiens, et le processus linéaire scolaire réside dans le rapport des actions aux contextes. Comme l'explique l'anthropologue Bazin (2008, p. 356) dans un texte d'épistémologie pratique, « je risque de fort mal raconter la bataille d'Austerlitz si je ne sais pas comment on fait la guerre à cette époque; mais pour apprendre comment on fait la guerre à cette époque la bataille d'Austerlitz peut me servir de leçon, pour autant que j'en ai un récit exact ». Chez le chercheur en sciences sociales, il n'y a pas de relation univoque entre connaissance du contexte pertinent et connaissance d'un événement singulier, exemplaire de ce contexte, mais des allers-retours multiples, des jeux d'échelles (Revel, 1996) entre étude de traces d'événements et généralisation. En classe, en revanche, le savoir déjà-là des élèves est peu mis en jeu. Non qu'il soit ignoré, mais il n'est généralement pas considéré comme suffisant pour jouer le rôle de "l'état des savoirs » chez les historiens. Dans l'exemple que nous avons pris, il est peu probable que les élèves de $4^{\text {ème }}$ ou de $2^{\text {nde }}$ aient quelque chose de pertinent à dire sur la manière dont des lecteurs des textes de Voltaire ont pu recevoir tel ou tel texte. Il est possible qu'ils puissent utiliser un modèle explicatif atemporel tel que «la lecture de ces idées de liberté et de justice ouvre les yeux sur la situation vécue », mais celui-ci ne pourrait s'ancrer sur des données spécifiques au XVIIIème siècle. Les connaissances potentielles des élèves en phase avec un tel modèle seraient sans doute celles d'un Ancien Régime marqué par une sujétion généralisée, mais qui ne dit rien de la période singulière de réception des textes en jeu.

Cet exemple illustre l'idée que l'absence de mise en jeu des connaissances des élèves sur le contexte de production et de réception des sources, qui est avéré par les travaux didactiques sur les pratiques ordinaires, serait épistémologiquement fondée : puisqu'ils ne sont pas en mesure d'interpréter le document dans son contexte, l'usage inductif qui en est fait apparait légitime. On peut cependant identifier des situations exceptionnelles qui contredisent cette généralisation didactique, et oblige à considérer les différences liées aux types de sources en jeu.

\section{La spécificité du témoignage et de son appréhension narrative}

Une séquence menée dans des classes de $\mathrm{CM} 2$ et de $3^{\text {ème }}$, sur la Première Guerre, repose sur l'étude de quelques documents liés à un soldat ayant réellement existét . Dans le fonctionnement ordinaire de la discipline scolaire, nous venons de le voir, les élèves instrumentalisent les documents pour construire un récit à partir des modèles de comportements humains qu'ils connaissent d'expérience. C'est ainsi qu'ils lisent la lettre que ce soldat adresse à sa famille : pour eux, elle ne fait que confirmer ce qu'ils savent déjà expliquer, que le soldat souffre et qu'il n'a pas le choix, qu'il est une victime. Les connaissances qu'ils mobilisent pour opérer l'interprétation de cette source concernent des données sur les guerres, et plus particulièrement celles qui supposent la mobilisation des citoyens en soldats (par opposition avec les guerres menées par des "guerriers", pour reprendre l'opposition formulée par le personnage du Capitaine Conan dans le roman de Vercel (1934), entre celui qui fait la guerre et celui qui la subit). Mais les élèves mobilisent aussi des modèles explicatifs spécifiques à l'interprétation du document que constitue la lettre: lorsqu'un individu est victime d'une situation sur laquelle il n'a pas prise, il s'en plaint. C'est ainsi, armés de modèles explicatifs, qu'ils sélectionnent dans la lettre les données concernant ce soldat. Ils en retiennent les confirmations de leurs 
modèles et de leur connaissance d'un contexte de guerre : le soldat écrit à sa famille les choses horribles qu'il voit et qu'il subit. Ils ignorent en revanche - tous les élèves de chacune des classes observées - la partie de la lettre qui contredit cette interprétation, lorsque le soldat écrit que le pire, ce ne sont pas les cadavres mais les destructions matérielles («j'ai déjà oublié les cadavres, mais ce que je n'oublierai jamais, c'est la ruine des choses, la destruction de maisons...", écrit-il à sa femme). La situation change cependant dans une des classes lorsque l'enseignante, en CM2, demande au cours de la mise en commun sur l'interprétation des traces : «ce soldat dit-il tout dans sa lettre?». Immédiatement, des élèves envisagent différemment la lettre. Elle n'est plus seulement un texte, elle est aussi une interaction entre le soldat et sa femme : il devient normal, aux yeux des élèves, qu'il fasse attention à ce qu'il écrit. Dans une version modifiée de la séquence, l'année suivante, nous organisons et planifions ce type de renversement notamment en soulevant explicitement le fait que la dernière partie, discordante, de la lettre est laissée dans l'ombre et qu'il faut tenter de l'expliquer. A nouveau, les élèves évoquent d'emblée des hypothèses explicatives en faisant appel à d'autres modèles de comportements que le récit et la plainte face à la situation : le soldat ne veut pas choquer sa famille, ne veut pas passer pour un assassin, veut la protéger... Plus encore, ils font émerger des contradictions qui posent un nouveau problème : il est étrange qu'il ne dise pas tout, car sa famille sait bien ce qu'il vit et ce qu'il fait, et elle sait aussi qu'il n'a pas le choix et qu'il le fait pour défendre son pays. Ce qui constitue les prémices d'un savoir tout à fait intéressant du point de vue historiographique : l'indicible de l'expérience de guerre et la coupure qui s'en suit entre le front et l'arrière. Voilà un savoir ignoré des élèves qui précise et développe la notion de victime à laquelle ils adhèrent au départ. Que se passe-til dans cette situation d'étude d'un témoignage, par comparaison avec l'analyse de la section précédente sur l'étude d'un texte philosophique?

Le récit qui se construit dans la classe avec la lettre du soldat est le récit de l'action que constitue cette trace du passé, alors que précédemment, le récit en jeu était celui de la conséquence univoque de la lecture d'un texte philosophique; d'un côté l'objet est la trace, de l'autre, c'est le passé lui-même. Les élèves appréhendent la lettre comme une interaction entre le soldat et sa femme parce qu'ils sont immédiatement capables de la considérer comme un témoignage adressé, sans doute parce qu'ils savent d'expérience ce qu'est cette situation (raconter ce qu'on a vécu est une expérience sociale courante, et avec elle, la précaution d'adapter son récit à son interlocuteur). Par contraste, le texte philosophique ne témoigne en rien de l'interaction avec ses lecteurs, c'est-à-dire qu'il ne fournit aux élèves aucune donnée qui leur permettrait de mettre en discussion le modèle explicatif qu'ils mobilisent pour comprendre l'action que constitue la publication du texte. Avec la lettre du soldat, le modèle explicatif de la plainte, conforté par une partie du texte, est au contraire mis en question par les données concernant la situation d'écriture du texte épistolaire, que les élèves appréhendent aisément.

On peut interpréter cette différence entre les deux dispositifs d'étude du passé par la spécificité du témoignage, dans le sens proposé par Ricœur (2000, p. 26) : le témoignage constitue une "structure fondamentale de transition entre la mémoire et l'histoire ». De ce fait, comme l'établit Fink (2014, p. 116) à propos des témoignages oraux, leur étude en classe présente un potentialité didactique unique : «Le processus de dénaturalisation (le témoin ne dit pas la réalité du monde) et d'analyse discursive et factuelle que requièrent les témoignages oraux en tant que source de connaissance et de compréhension du passé sont l'occasion de travailler les modes de construction et de pensée en histoire ». Une 
telle potentialité s'explique par la nature singulière du témoignage qui le rattache anthropologiquement à l'expérience sociale de chacun, par le biais de la narration. Ricœur (2000, p. 203) contraste ainsi le témoignage avec les archives: «l'activité de témoigner, saisie en deçà de la bifurcation entre son usage judiciaire et son usage historiographique, révèle alors la même ampleur et la même portée que celle de raconter en vertu de la parenté manifeste entre les deux activités ».

L'appréhension par les élèves, dans le deuxième temps de la séquence, de la lettre du soldat comme d'une situation d'action (d'échange épistolaire) leur permet de raconter ce qu'il fait : il écrit à sa femme pour lui donner des nouvelles, mais ces nouvelles peuvent apparaître traumatisantes par la radicalité de l'expérience vécue, il adapte donc son témoignage à ce qu'il pense être acceptable pour sa femme. Ce récit court mais dense fait cas dans la classe par rapport au savoir initialement établi sur ce que la lettre dit de l'expérience du combat (le soldat témoigne de sa souffrance de victime). Il fait événement dans la classe, au sens de Revel (2001, p. 105), c'est-à-dire qu'il «donne accès, ou plus simplement, il autorise un point de vue sur quelque chose qui le dépasse et qui n'a pas de mesure commune avec lui »: la lettre donne accès, aujourd'hui, à des dimensions inconnues et non exprimées par les acteurs eux-mêmes, de l'expérience des tranchées. On peut l'interpréter comme un processus de problématisation dans la mesure où cette remise en question de l'explication initiale oblige à poursuivre l'enquête dans une nouvelle direction. Il ne s'agit plus seulement de corroborer l'idée explicative d'un rôle de victime du soldat souffrant de la peur et de l'horreur vécue, mais d'expliquer pourquoi il ne peut en parler à l'arrière.

\section{Le récit de l'enquête dans la temporalité de la classe}

La comparaison des deux situations d'enseignement peut être interprétée par le biais d'un retour à une des caractéristiques épistémologiques principales de l'écriture scientifique du passé. Contrairement au récit de sens commun sur le passé, le récit d'historien pour les historiens mêle explication de l'événement et narration de l'enquête, ce qui produit les raisons d'accorder crédit aux explications de l'événement. Comme le suggère l'historien de l'histoire Grafton (1998, p. 174), «l'histoire narrative a formellement "bougé" en permanence dans les derniers siècles, et [...] elle a évolué parce que les historiens n'ont cessé de chercher de nouvelles manières de dire l'histoire de leurs recherches aussi bien que l'histoire des objets dont ils traitaient, à deux niveaux séparés et dans des temps différents ». Il semble que ce double récit n'a pas de sens dans le cas de l'étude scolaire des textes des philosophes des Lumières, alors qu'il en a dans le cas de l'étude de la lettre du soldat.

En effet, dans le premier cas, l'enquête menée par les élèves dans le cadre des activités habituelles de la classe - lecture des documents, réponse à des questions de sélection d'information et de reproduction de savoirs antérieurs, production d'une explication - ne constitue qu'une mise en ordre chronologique et causale d'une succession d'événements. Le temps de la classe ne se distingue pas de la recherche du temps de l'événement parce que l'usage du modèle explicatif atemporel n'est pas mis en discussion par le document, et parce que le temps naturel de la chronologie garantit la cohérence de la narration. L'étude se déroule comme d'habitude dans le cours de la classe d'histoire, données et modèles s'accordent dans une explication qui est nécessairement conforme aux attentes parce que chronologique. En revanche, dans le cas de l'étude de la lettre du soldat, le 
temps de l'établissement de l'explication conforme (première phase de la séquence), et le temps de sa remise en cause (deuxième phase), organisent une rupture, un événement, qui n'a de sens qu'à l'échelle de l'activité de la classe et de son histoire propre. L'histoire des recherches dans la classe est donc non seulement une mise en cohérence dans une intrigue acceptable qui est celle de la causalité chronologique, mais elle offre simultanément une intrigue intrigante (au sens de Baroni, 2010). Ainsi dans une classe de troisième le professeur fait-il explicitement travailler ses élèves sur le cas du soldat en posant la question de la valeur du travail fourni jusque là : nous avons mal lu cette lettre en laissant de côté la fin, dit-il ; celle-ci contredit notre explication; comment alors expliquer cette contradiction ? Le comportement du soldat écrivant à sa femme s'obscurcit pour les élèves, la lecture de sa lettre devient intrigante alors qu'elle n'offrait précédemment qu'une confirmation de ce qu'on pensait de sa situation.

On identifie par ce biais, mais pour l'histoire de la recherche dans la classe, ce que Baroni (2010) qualifie de tension narrative dans le texte du roman, à partir de l'idée de configuration proposée par Ricœur. La fonction intrigante de l'enquête que raconte le professeur dans le fil de la séquence n'a de sens qu'en relation avec la fonction configurante de l'enquête habituellement menée. La contradiction dans le texte même de la lettre ou bien la question de savoir si le soldat dit tout dans sa lettre met en crise ce processus configurant propre aux habitudes disciplinaires scolaires. Mais c'est bien parce que ce processus est intégré par les élèves comme permettant de produire des explications cohérentes sur le passé que la question du professeur ou la contradiction de la lettre devient intrigante pour les enquêteurs que sont les élèves.

On entrevoit par là les conditions de possibilité d'un double récit, de l'objet et de l'enquête, dans la classe d'histoire analogue à celui des historiens dans leur communauté. La seule mise en ordre chronologique des événements à partir des sources ne permet en effet pas de distinguer un récit de l'autre, comme dans le cas des textes de philosophes et de la Révolution. En revanche, la production, volontaire ou non, d'un événement dans le déroulement normal de l'étude de documents dans la classe constitue l'activité de recherche en récit digne d'être raconté. Il devient pertinent parce qu'il intrigue par rapport à la logique d'explication de l'événement. Il se passe dans la classe quelque chose dont il devient intéressant de témoigner dans la trace écrite finale.

\section{Conclusion}

31 La perspective narrative engagée ici pour appréhender certains enjeux didactiques de l'enseignement de l'histoire fait émerger la place essentielle que peuvent jouer les témoignages dans le travail scolaire sur le passé. Son rôle transitionnel suggéré par Ricœur peut en effet s'entendre dans une dimension scolaire que l'enseignant est susceptible de déployer. Cette potentialité pragmatique s'ancre dans les principes épistémologiques élaborés au fil des siècles pour organiser et cadrer les pratiques historiennes à même de dépasser la linéarité d'un usage strictement causal des traces. Par ailleurs, cette potentialité développementale du témoignage réside dans sa nature intrinsèquement narrative qui constitue une porte d'entrée de l'expérience mémorielle partagée par tout individu, vers le travail formel et systématique de l'histoire. Elle rend possible le jeu d'échelles entre l'expérience de l'élève aujourd'hui, et l'expérience passée du témoin, par le biais de la mise au travail des interactions entre individu et société. Interactions qui portent aussi bien sur les relations entre acteurs de l'événement passé 
que sur les relations entre acteurs présents de l'histoire de la recherche dans la classe, par l'intermédiaire des événements qui la jalonnent.

\section{BIBLIOGRAPHY}

Baroni, R. (2010). Le temps de l'intrigue. Cahiers de Narratologie. Analyse et théorie narratives, (18).

Bazin, J. (2008). Des clous dans la Joconde : l'anthropologie autrement. Toulouse, France: Anacharsis.

Bronckart, J.-P. (1996). Activité langagière, textes et discours. Pour un interactionisme sociodiscursif. Lausanne : Delachaux et Niestlé.

Chartier, R. (2000). Les origines culturelles de la Révolution française (Vol. 268). Paris : Seuil.

Doussot, S. (2011). Didactique de l'histoire : outils et pratiques de l'enquête historienne en classe. Rennes, France : Presses universitaires de Rennes.

Doussot, S., \& Vézier, A. (2015). Débat historiographique sur le Grande Guerre et étude de cas en histoire : des références pour la classe ? Didactica Historica, 1, 83-88.

Fabre, M. (2009). Philosophie et pédagogie du problème. Paris : Vrin.

Fink, N. (2014). Paroles de témoins, paroles d'élèves. La mémoire et l'histoire de la Seconde Guerre mondiale, de l'espace public au monde scolaire. Berne : Peter Lang.

Gerin-Grataloup, A.-M., Solonel, M., \& Tutiaux-Guillon, N. (1994). Situations-problèmes et situations scolaires en histoire-géographie. Revue française de pédagogie, 106(1), 25-37. https:// doi.org/10.3406/rfp.1994.1270

Ginzburg, C. (2008). Préface. Preuves et possibilités. In Le retour de Martin Guerre (N. Z. Davis, p. 9-43). Paris : Taillandier.

Grafton, A. T. (1998). Les origines tragiques de l'érudition une histoire de la note en bas de page. (P.-A. Fabre, Trad.). Paris : Seuil.

Levisohn, J. A. (2010). Negotiating historical narratives : An epistemology of history for history education. Journal of Philosophy of Education, 44(1), 1-21.

Orange, C. (2005). Problématisation et conceptualisation dans les sciences et dans les apprentissages scientifiques. Les sciences de l'éducation - Pour l'ère nouvelle, 38(3), 69-93.

Revel, J. (1996). Jeux d'échelles : la micro-analyse à l'expérience. Paris : Seuil.

Revel, J. (2001). Retour sur l'événement : un itinéraire historiographique. In Le goût de l'enquête : pour Jean-Claude Passeron (J.-L. Fabiani, p. 95-118). Paris : L'harmattan.

Ricœur, P. (2000). La mémoire, l'histoire, l’oubli. Paris : Éd. du Seuil.

Tutiaux-Guillon, N. (2008). Interpréter la stabilité d'une discipline scolaire : l'histoire-géographie dans le secondaire français. In Compétences et contenus. Les curriculum en question (p. 117-146). Bruxelles : De Bœck. 
Tutiaux-Guillon, N. (2009). L'histoire scolaire au risque des sociétés en mutation. Raisons Comparaisons Educations. La revue française d'éducation comparée, (4), 105-118.

Vercel, R. (1934). Capitaine Conan. Paris : Albin Michel.

White, H. (1978). Tropics of discourse. Philadelphia : Johns Hopkins University Press.

Wineburg, S. (2001). Historical thinking and other unnatural acts : charting the future of teaching the past (Vol. 1- 1). Philadelphia : Temple University Press.

\section{NOTES}

1. Au sein de l'équipe didactique du laboratoire du CREN (Centre de recherche en éducation de Nantes) dans le séminaire "problématisation ", sur la base des travaux fondateurs de Fabre (2009) et Orange (2005).

2. La seconde attente principale est de prélever des informations dans les documents (TutiauxGuillon, 2009).

3. Cette élève de CM1 est dans une classe de double niveau CM1-CM2, et elle a écouté la leçon d'histoire des CM2.

4. Une description détaillée de la séquence et de ses enjeux didactiques est fournie dans Doussot \& Vézier (2015).

\section{ABSTRACTS}

Despite the fact that historians' argumentation lays on narration, their narratives come from inquiries that differ from other practices. Comparing this specific relation between historical inquiry and historical texts highlights how difficult it is for history classrooms to link document practices and production of argued narratives. The multiplicity of narratives and points of view brought in the classroom through documents, schoolbooks, teachers and pupils can be handled in different ways. Two examples show two different learning potentialities of historical argumentation. The one that uses testimonies might play an essential part as a transition between the social experience of the pupils, and the idea of criticism in scientific history.

Si l'argumentation des historiens repose sur la narration, leurs récits sont issus d'enquêtes qui se démarquent d'autres pratiques de production de récits. C'est par comparaison avec ce rapport de l'enquête au texte des historiens que l'histoire scolaire rencontre des difficultés à associer pratiques documentaires et production de récits argumentés. La multiplicité des récits et des points de vue portés par les documents, les manuels et les acteurs de la classe (enseignant et élèves) peut être gérée de différentes manières. Deux exemples illustrent des potentialités différentes d'apprentissage des spécificités de l'argumentation historique. Parmi elles, celles portées l'étude des témoignages se révèlent essentielles par le rôle transitionnel qu'elles peuvent jouer entre l'expérience sociale des élèves et les visées critiques de l'histoire scientifique. 
INDEX

Mots-clés: histoire, narration, problématisation, témoignage

\section{AUTHOR}

\section{SYLVAIN DOUSSOT}

ESPE - CREN (Université de Nantes), 4 chemin de Launay-Violette, 44300 Nantes,

sylvain.doussot@univ-nantes.fr 